\title{
Adsorption of Polyelectrolyte Solutions on Surfaces: A Debye-Hückel Theory
}

\author{
Xavier Châtellier and Jean-François Joanny $\left(^{*}\right)$ \\ Institut Charles Sadron, 6 rue Boussingault, 67083 Strasbourg, France
}

(Received 3 July 1996, received in final form 6 September 1996, accepted 9 September 1996)

PACS.05.90. $+\mathrm{m}$ - Other topics in statistical physics and thermodynamics

PACS.82.65.Dp - Thermodynamics of surfaces and interfaces

PACS.82.70.Dd - Colloids

\begin{abstract}
The adsorption onto a solid surface of a flexible polvelectrolyte solution in equilibrium with a reservoir is investigated. Both the electrostatic and the short-ranged interactions with the interface are taken into account. The polyelectrolyte chains are supposed to be infinitely long and to obey Gaussian statistics. We obtain analytical results for the concentration profiles within the framework of a mean-field theory and in the limit of weak adsorption (linear approximation). At high salt concentration, the polyelectrolyte chains behave as neutral polymers and electrostatic interactions are screened over the Debye-Hückel length. At low salt concentration, the profiles show damped oscillations. Similarly, the energy and the force between two interfaces mediated by the polyelectrolyte solution can show oscillations as a function of the separation between the interfaces. At the onset of the instability of the bulk polyelectrolyte solution, the structure factor diverges at the wavevector of the oscillations. We also consider the polymer surface excess and find a condition for which it shows a maximum at a given value of the fraction of charged monomers.
\end{abstract}

\section{Introduction}

Colloidal stabilization and flocculation are important for numerous applications, ranging from waste water treatment to various industrial processes in food technology or paint production. They have been studied quite extensively over the last years. Polymers are widely used in colloidal systems, as they can interact with the constituents and act both as stabilizing and destabilizing agents. Their effect results from a subtle balance between the various interactions in the system and a good control of colloidal stabilization requires very precise experimental conditions [1]. For instance, when the polymer adsorbs on the surface of colloidal particles, flocculation can be induced by a bridging process, or on the contrary, stability may be enhanced because of steric repulsions between adsorbed polymer layers. The adsorption of neutral polymers has been studied in details, experimentally as well as theoretically, and a good understanding of these effects has been reached [2]. It is generally accepted that the conformations of the adsorbed polymer chains are decisive in determining the dominant effect, and that steric stabilization is enhanced by a strong irreversible adsorption, that leads to less penetrable adsorbed layers.

(*) Author for correspondence (e-mail: joanny@ics-crm.u-strabg.fr) 
Polyelectrolytes are charged polymers and have thus specific properties, due to the longrange character of the electrostatic interactions. The repulsion between the charged monomers and the loss of entropy of counter-ions in a phase separation usually leads to a better solubility, e.g. in water; this opens new possibilities for designing less polluting materials, a common example being water-soluble paints. The adsorption of polyelectrolyte chains is hindered by the repulsion between the monomers, which prevents the build up of a dense adsorbed layer. In addition to the short-range van der Waals attractions responsible for the adsorption of neutral polymers, an electrostatic interaction with the interface can also strongly influence the adsorption. The electrostatic interactions in a polyelectrolyte solution can be modulated by changing the ionic strength by addition of salt. They are then screened over a mesoscopic length scale (the Debye-Hückel length) which decreases when the salt concentration increases, and the behavior of charged polymer solutions tends to that of neutral ones at high salt concentrations. At lower concentrations, the variation of the ionic strength has a rather intricate effect on polyelectrolyte adsorption, since both the electrostatic interaction between the charged monomers and the interface and the repulsion between the monomers are screened. All these elements being taken into account, a richer variety of behaviors is expected for the adsorption of charged polymers than for that of neutral polymers. In particular, more complex stabilization or flocculation processes can occur, where the total charge of a colloidal particle and its adsorbed polymer layer leads to an electrostatic repulsion between particles (or, on the contrary, when combined to van der Waals forces, to attractions if the charge is neutralized).

Many experiments have been performed on the adsorption of polyelectrolyte chains at interfaces and have emphasized the complexity of the adsorption process. Some theoretical work has also been carried out. But, because of the many parameters involved, a satisfying understanding is still lacking. The early approaches assumed non-realistic monomer concentration profiles [3]. More recently, the self-consistent field method of Scheutjens-Fleer has been adapted to include long-ranged interactions; however, it still relies on numerical determinations of the concentration profiles and of the adsorbed polymer amount. Polyelectrolyte adsorption has also been studied using mean-field approximations for the free energy that lead to the PoissonBoltzmann equation [4]. These equations were then solved numerically. In all these mean field approaches, it is implicitly assumed that the polymer chains conformation remains Gaussian. In dilute solutions, the electrostatic interactions between monomers are strong and tend to stiffen the chain locally. This effect is not taken into account by the mean-field theory but becomes small at large enough concentrations [5].

In this paper we adopt the mean-field approach [4] but we focus on the weak adsorption region where analytical results can be obtained. Bulk polyelectrolyte solutions have already been studied using the same approximation. An instability at non-zero wavevector is predicted if the salt concentration is low enough $[6,7]$. In Section 2, we introduce the free energy and discuss the stability of a bulk polyelectrolyte solution. The adsorption of a polyelectrolyte solution at a single interface is considered in Section 3. In the linear approximation, which corresponds to small interactions between the polymer and the interface, the equations can be solved analytically. This allows to give a more qualitative description of the adsorption in the various regions of the phase diagram. We obtain explicit expressions for the concentration profiles and the interaction between charged surfaces (colloidal particles) mediated by a polyelectrolyte solution (Sect. 4). In some cases, the concentration profiles show oscillations. This leads to a simple form for the polymer surface excess, which gives an estimate for the total adsorbed charge (Sect. 5). In the last Section 6, the assumptions and the limits of the model are discussed. 


\section{Free Energy of an Inhomogeneous Polyelectrolyte Solution}

In this section, the free energy of an inhomogeneous polyelectrolyte solution introduced by Borue and Erukhimovich [6] and Joanny and Leibler [7] is discussed. The free energy contributions accounting for the interaction with the surface are introduced in the next section. In a continuous model, the mean-field free energy $F$ of the polyelectrolyte solution is written as an integral over space of the free energy density, and has several contributions.

$$
F=F_{\text {ent }}+F_{\text {pol }}+F_{\text {elec }}
$$

$F_{\text {ent }}$ is an entropic part due to the counter-ions and to the salt molecules. A technical simplification assumes that the polyelectrolyte and the salt have the same counter-ion, and that charged monomers, salt and counter-ion molecules have the same valency $Z$. We consider the situation of a solution in contact with a reservoir where the concentrations of monomers, salt and counter-ion are respectively $\phi_{\mathrm{b}}, n_{\mathrm{b}}$ and $\phi_{\mathrm{ib}}$. The overall electroneutrality in the reservoir imposes that $f \phi_{\mathrm{b}}+n_{\mathrm{b}}-\phi_{\mathrm{ib}}=0$, where $f$ is the fraction of charged monomers on a chain. In the following, we assume that the charges are quenched along the chain i.e. that $f<1$ is a fixed and known number. The reservoir imposes the chemical potential of the various components and the relevant free energy is the grand canonical free energy (at constant chemical potentials). The free energy $F_{\text {ent }}$ is expressed as

$$
F_{\text {ent }}=k_{\mathrm{B}} T \int \mathrm{d} \mathbf{r}\left[\phi_{\mathrm{i}}(\mathbf{r}) \log \frac{\phi_{\mathrm{i}}(\mathbf{r})}{\phi_{\mathrm{ib}}}-\left(\phi_{\mathrm{i}}(\mathbf{r})-\phi_{\mathrm{ib}}\right)+n(\mathbf{r}) \log \frac{n(\mathbf{r})}{n_{\mathrm{b}}}-\left(n(\mathbf{r})-n_{\mathrm{b}}\right)\right]
$$

$T$ is the temperature and $k_{\mathrm{B}}$ is the Boltzmann constant. As can be seen from equation (2), $F_{\text {ent }}$ is minimized when the concentrations of salt and counter-ions are constant throughout the solution and equal to their values in the reservoir (by convention, $F_{\text {ent }}=0$ when $\phi_{1}(\mathbf{r})=\phi_{1 \mathrm{~b}}$ and $n(\mathbf{r})=n_{\mathrm{b}}$ ).

$F_{\text {pol }}$ is the polymer contribution to the free energy. In a self-consistent mean-field model developed first by Edwards [8], where the statistics of polymer chains are assumed to be Gaussian and where the chains are infinitely long (neglecting thus the translational entropy of the chains),

$$
F_{\mathrm{pol}}=k_{\mathrm{B}} T \int \mathrm{d} \mathbf{r}\left[\frac{a^{2}}{24 \phi(\mathbf{r})}|\nabla \phi|^{2}(\mathbf{r})+\frac{1}{2} \tilde{v}\left(\phi(\mathbf{r})-\phi_{\mathrm{b}}\right)^{2}\right]
$$

$a$ is the size of a monomer and $\tilde{v}^{\prime} \equiv v+w^{2}\left(\phi(\mathbf{r})+2 \phi_{\mathrm{b}}\right) / 3$ is the effectrve excluded volume parameter, where $v$ and $w^{2}$ are respectively the second and the third virial coefficients. The excluded volume $v$ is of order $a^{3}$ and is either positive (in a good solvent) or negative (in a poor solvent); most polyelectrolytes are organic polymers and are thus in a poor solvent in water. The third virial coefficient $w^{2}$, of order $a^{6}$, is supposed here to be positive; it stabilizes the solution in a poor solvent. A neutral polymer solution is unstable if $\tilde{v}<0$. When $\tilde{v}>0$, the Edwards correlation length $\xi$ is defined as $\xi^{2} \equiv a^{2} /\left(3 \tilde{v} \phi_{\mathrm{b}}\right)$. This is the length scale that characterizes the spatial relaxation of concentration fluctuations and for example, the concentration profile of a neutral polymer solution adsorbing at an interface relaxes towards its bulk value over a typical length scale $\xi$ [9]. The first term in equation (3) accounts for the elasticity of the polymer chains and the second term describes the interactions between the monomers, and the chemical potential terms due to the equilibrium with the reservoir. $F_{\text {pol }}$ is the difference between the grand canonical energies of a polymer solution with concentration profile $\phi(\mathbf{r})$ and of an homogeneous solution of concentration $\phi_{\mathrm{b}}$.

The electrostatic free energy is

$$
F_{\text {elec }}=\frac{1}{2} \int \mathrm{d} \mathbf{r} \varepsilon E^{2}(\mathbf{r})
$$


where $\varepsilon$ is the dielectric constant of the solution (typically $\varepsilon \approx 80$ for water) and $\mathbf{E}(\mathbf{r})$ is the electric field at point $\mathbf{r}$. A typical parameter for estimating the strength of electric forces in a medium is the Bjerrum length, at which the Coulomb interaction between two charges is equal to $k_{\mathrm{B}} T, l_{\mathrm{B}} \equiv(Z e)^{2} /\left(4 \pi \varepsilon k_{\mathrm{B}} T\right)$, where $e$ is the elementary electric charge, and $Z$ the valency of the interacting charges. For monovalent ions in water at room temperature, $l_{\mathrm{B}} \approx 7 \AA$. We suppose, without loss of generality, that the charged monomers are negatively charged. The local charge density $\rho=-e\left[f \phi(\mathbf{r})+n(\mathbf{r})-\phi_{\mathbf{1}}(\mathbf{r})\right]$ is related to the electric field by Poisson equation $\operatorname{div} \mathbf{E}=\rho / \varepsilon$.

In a bulk solution, in contact with the reservoir, the concentrations are at any point equal on average to the reservoir values. However there are spatial concentration fluctuations. In the following we use, instead of $\phi(\mathbf{r}), \phi_{\mathrm{i}}(\mathbf{r})$ and $n(\mathbf{r})$, the fluctuation variables $\delta n(\mathbf{r}) \equiv n(\mathbf{r})-n_{\mathrm{b}}$, $\delta \phi_{\mathrm{i}}(\mathbf{r}) \equiv \phi_{\mathrm{i}}(\mathbf{r})-\phi_{\mathrm{ib}}$ and $\delta \phi(\mathbf{r}) \equiv \phi(\mathbf{r})-\phi_{\mathrm{b}}$ and their Fourier transforms. The limit of small fluctuations is defined as $\delta n(\mathbf{r}) \ll n_{\mathrm{b}}, \delta \phi_{1}(\mathbf{r}) \ll \phi_{1 \mathrm{~b}}$ and $\delta \phi(\mathbf{r}) \ll \phi_{\mathrm{b}}$. In this limit the free energy is approximated by

$$
F=\frac{1}{2} k_{\mathrm{B}} T \int \mathrm{d} \mathbf{r}\left[\frac{\delta \phi_{1}^{2}(\mathbf{r})}{\phi_{\mathrm{ib}}}+\frac{\delta n^{2}(\mathbf{r})}{n_{\mathrm{b}}}+\frac{a^{2}}{12 \phi_{\mathrm{b}}}|\nabla \delta \phi|^{2}(\mathbf{r})+\tilde{v} \delta \phi^{2}(\mathbf{r})\right]+F_{\text {elec }}
$$

The free energy is conveniently expressed in terms of the Fourier components of the concentrations

$$
F=\frac{1}{2} k_{\mathrm{B}} T \sum_{\mathbf{q}} \delta \Phi_{\mathbf{q}} S^{-1}(\mathbf{q}) \cdot \delta \Phi_{-\mathbf{q}}
$$

where $\delta \Phi_{\mathbf{q}} \equiv\left(\delta \phi_{\mathbf{q}}, \delta n_{\mathbf{q}}, \delta \phi_{\mathrm{iq}}\right)$ denotes the three-dimensional vector defining a state of the system, and $S^{-1}(\mathbf{q})$ is the inverse structure factor $3 \times 3$-matrix.

$$
S^{-1}(\mathbf{q})=\frac{a^{5}}{12 \phi_{\mathrm{b}} q^{2}}\left(\begin{array}{ccc}
q^{4}+\left(\frac{12 \tilde{v} \phi_{\mathrm{b}}}{a^{2}}\right) q^{2}+q^{* 4} & \frac{q^{* 4}}{f} & -\frac{q^{* 4}}{f} \\
\frac{q^{* 4}}{f} & \frac{12 q^{2} \phi_{\mathrm{b}}}{n_{\mathrm{b}} a^{2}}+\frac{q^{* 4}}{f^{2}} & -\frac{q^{* 4}}{f^{2}} \\
-\frac{q^{* 4}}{f} & -\frac{q^{* 4}}{f^{2}} & \frac{12 q^{2} \phi_{\mathrm{b}}}{\phi_{\mathrm{lb}} a^{2}}+\frac{q^{* 4}}{f^{2}}
\end{array}\right)
$$

The characteristic wavevector $q^{*}$ is defined by $q^{* 4} \equiv 48 \pi l_{\mathrm{B}} \phi_{\mathrm{b}} f^{2} / a^{2}$. The importance of the thermal fluctuations at a given wavevector $q$ can be estimated by studying the monomermonomer correlation function $\left\langle\delta \phi_{\mathrm{q}} \delta \phi_{-\mathrm{q}}\right\rangle$ (where 〈〉 denotes a statistical average), obtained by inversion of the matrix $S^{-1}(\mathbf{q})$ and first calculated in references $[6,7]$

$$
\left\langle\delta \phi_{\mathbf{q}} \delta \phi_{-\mathbf{q}}\right\rangle=\frac{12 \phi_{\mathrm{b}}}{a^{5}} \frac{q^{2}+\kappa^{2}}{\left(q^{2}+\kappa^{2}\right)\left(q^{2}+12 \tilde{v} \phi_{\mathrm{b}} / a^{2}\right)+q^{* 4}}
$$

where $\kappa^{-1}$ is the Debye-Hückel length, defined as $\kappa^{2} \equiv 4 \pi l_{\mathrm{B}}\left(n_{\mathrm{b}}+\phi_{\mathrm{ib}}\right)$.

The phase diagram of the solution is displayed in Figure la. Instead of the variables $n$ (salt concentration), $\tilde{v}$ (temperature or excluded volume) and $\phi$, we use $q^{* 2} / \kappa^{2}$ and $12 \tilde{v} \phi_{\mathrm{b}} /(\kappa a)^{2}$ as variables. At a fixed concentration $\phi_{\mathrm{b}}$ and a fixed total ionic strength $\kappa^{2}$, the two axes can be thought of as the excluded volume $\tilde{v}$ and the fraction of charged monomers $f$. Note also that if the effective excluded volume $\tilde{v}$ is positive, $12 \tilde{v} \phi_{\mathrm{b}} / a^{2}=4 / \xi^{2}$ where $\xi$ is the correlation length of the equivalent neutral polymer solution (in the absence of charges). We do not calculate here the precise phase boundaries but we show the region of instability of a polyelectrolyte solution predicted bv the model. Because of the global electroneutrality condition, $f \phi_{\mathrm{b}}=\phi_{\mathrm{b}}-n_{\mathrm{b}}$, 


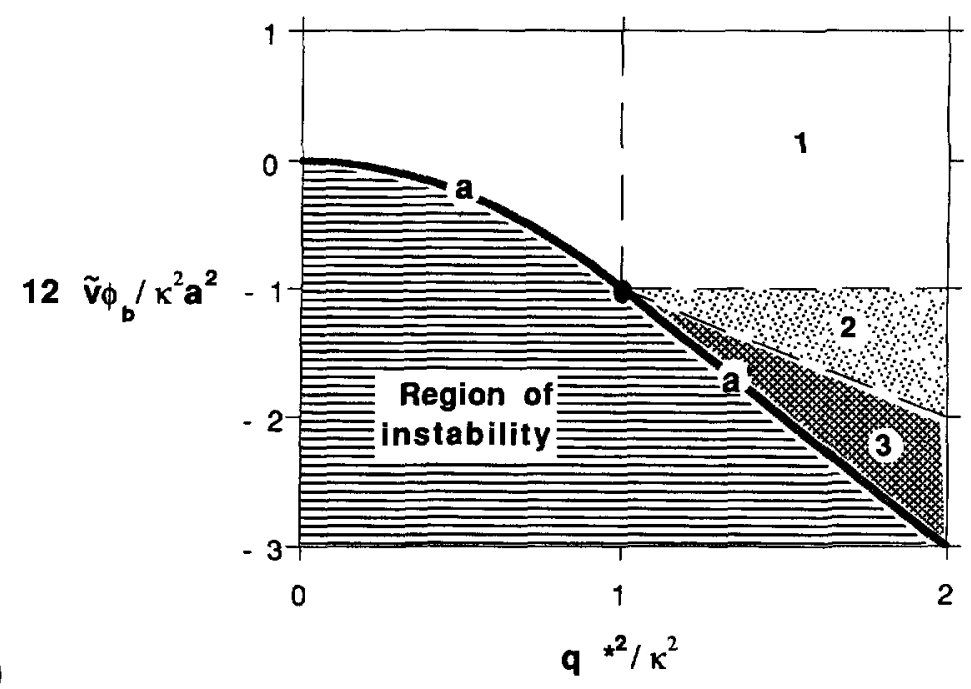

a)

$q \star^{2} / \kappa^{2}$

b)

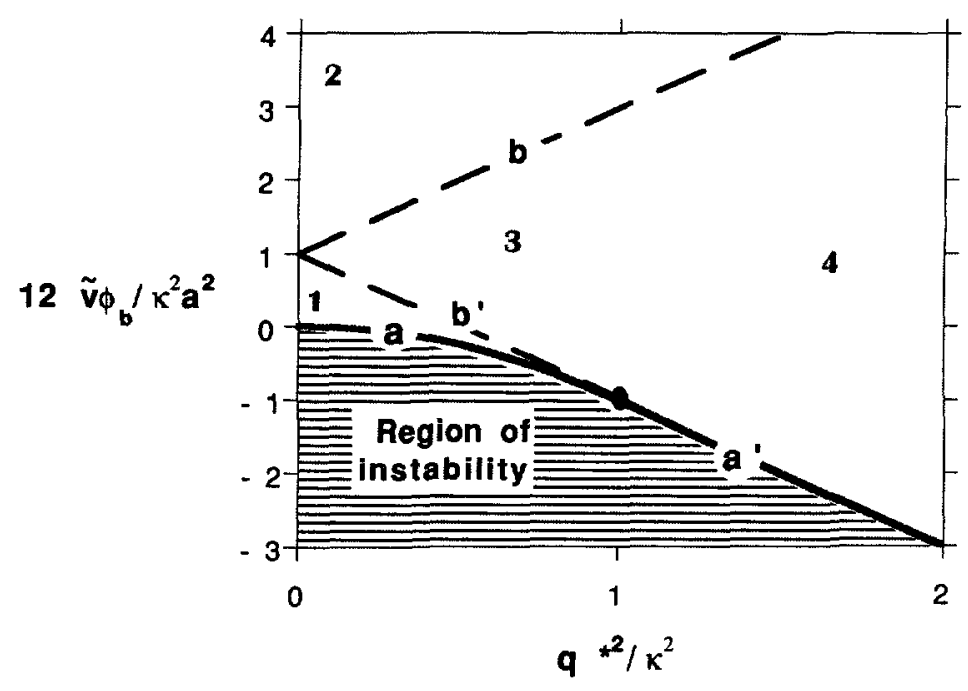

Fig. 1. - Diagram of stability of the bulk polyelectrolyte solution, in terms of the parameters $12 \tilde{v} \phi_{\mathrm{b}} /\left(\kappa^{2} a^{2}\right)$ and $q^{* 2} / \kappa^{2}$.

there is, at a given value of the fraction of charged monomers $f$. a minimal value of $\kappa$ (or, equivalently, there is a maximal value allowed for $q^{*}$ when $\kappa$ is fixed); it is achieved when there are only counter-ions in the solution (no salt) and corresponds to $q^{*}=\kappa\left(\pi \phi_{\mathrm{b}} l_{\mathrm{B}} a^{2} / 3\right)^{-1 / 4}$ When $q^{*}>\kappa$ (regions 1, 2 and 3 in Fig. 1a), the monomer-monomer correlation function has a peak at a wavevector $q_{\mathrm{m}}$ such as $q_{\mathrm{m}}^{2}=q^{* 2}-\kappa^{2}$, and the structure factor diverges when the effective excluded volume is decreased to $\tilde{v}=-\left(2 q^{* 2}-\kappa^{2}\right) a^{2} /\left(12 \phi_{\mathrm{b}}\right)$ (line $\mathrm{a}^{\prime}$ in Fig. 1). Hence $q^{*}$ can be interpreted as the wavevector at which the structure factor diverges at very low ionic strength $\left(\kappa \ll q^{*}\right)$. The divergence of the structure factor at non-zero wavevector has been discussed in references $[6,7]$; it indicates an instability of the solution at a finite wave vector that leads to the formation of mesophases. Qualitatively, it results from a non-trivial 


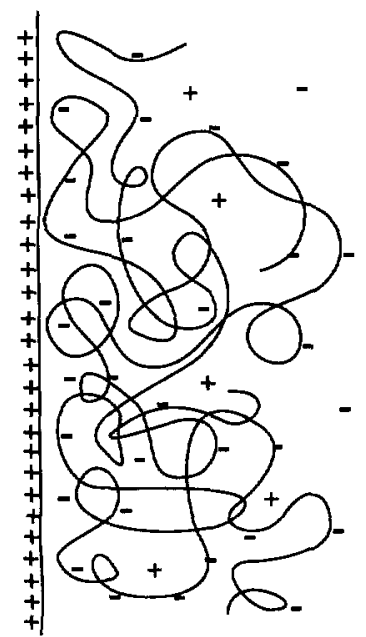

a)

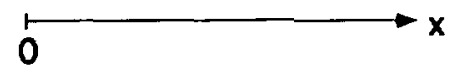

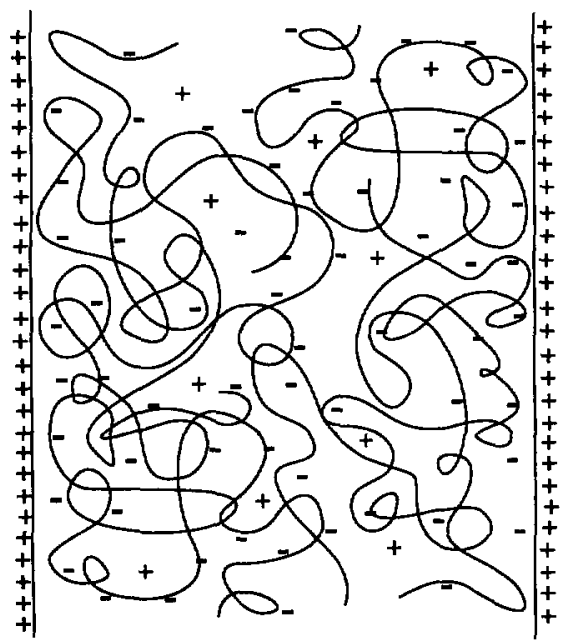

b)

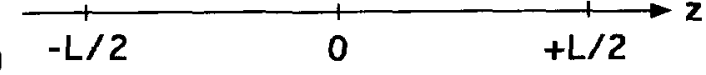

Fig. 2. - Schematic representation of the adsorption of a solution of flexible polyelectrolyte chains a) at a single interface or b) between two surfaces. The surfaces are supposed to be perfectly flat and homogeneous, with a positive charge.

interplay between the tendency of the polymer chains to collapse at macroscopic scales (but, in this case, the overall electroneutrality imposes that the ions are captured, at least partly, in the collapsed phase) and the gain in entropy for the ions if mesophases are formed, where the electroneutrality can be locally violated. When $q^{*}<\kappa$, the peak of the structure factor occurs at zero wavevector and the instability at $\tilde{v}=-a^{2} q^{* 4} /\left(12 \kappa^{2} \phi_{\mathrm{b}}\right)$ is the signature of a macroscopic phase separation between polymer and solvent (line a in Fig. 1). The two lines of instability join at a Lifshitz point [7].

The other correlation functions are also obtained from the matrix $S(\mathbf{q})$. It can easily be shown that the monomer-ion correlation functions $\left\langle\delta \phi_{\mathbf{q}} \delta n_{-\mathbf{q}}\right\rangle$ and $\left\langle\delta \phi_{\mathbf{q}} \delta \phi_{\mathrm{i}-\mathbf{q}}\right\rangle$ have a peak at non-zero wavevector $q_{\mathrm{i}}$, with $q_{\mathrm{i}}^{2}=-\left(\kappa^{2}+12 \tilde{v} \phi_{\mathrm{b}} / a^{2}\right) / 2$, when $\tilde{v}<-\kappa^{2} a^{2} /\left(12 \phi_{\mathrm{b}}\right)$ (regions 2 and 3 in Fig. 1a). And the ion-ion correlation functions $\left\langle\delta n_{\mathbf{q}} \delta \phi_{1-\mathbf{q}}\right\rangle,\left\langle\delta n_{\mathbf{q}} \delta n_{-\mathbf{q}}\right\rangle$ and $\left\langle\delta \phi_{\mathrm{iq}} \delta \phi_{\mathrm{i}-\mathrm{q}}\right\rangle$ have a peak at non-zero wavevector $q_{\mathrm{n}}$. such that $q_{\mathrm{n}}^{2}=-q^{* 2}-12 \tilde{v} \phi_{\mathrm{b}} / a^{2}$ when $\tilde{v}<-q^{* 2} a^{2} /\left(12 \phi_{\mathrm{b}}\right)$ (region 3 in Fig. 1a). At the onset of instability, all correlation functions diverge at the same wavevector, $q_{\mathrm{m}}^{2}=q^{* 2}-\kappa^{2}$.

\section{Interaction of a Polyelectrolyte Solution with a Single Ideal Interface}

3.1. The Debye-Hückel Equations. - In this section, we consider a polyelectrolyte solution with added salt interacting with a single, infinite, ideal (namely perfectly flat and chemically homogeneous) and charged interface. The distance of a point in the polyelectrolyte solution from the interface is denoted by $x>0$ (see Fig. 2a). For negative values of $x$, we suppose that the electric potential is constant $\left(E_{x}(x<0)=0\right)$. The surface charge per unit area is denoted by $\sigma$. Maxwell equations impose that the electric field at $x=0^{+}$is $E_{x}\left(x=0^{+}\right)=\sigma / \varepsilon$, where $\varepsilon$ is the dielectric constant of the solution; in a first approximation, $\varepsilon$ is supposed to be independent of the concentrations of ions and monomers and equal to the dielectric constant 
of the pure solvent; for water, $\varepsilon \approx 80$. The monomers also interact with the surface via a short range non-electrostatic interaction. The adsorption free energy $F_{\text {sr }}$ can be expanded to first order in the monomer concentration,

$$
F_{\mathrm{sr}}=-k_{\mathrm{B}} T \gamma \int \mathrm{d} \mathbf{r} \delta \phi(\mathbf{r}) a \delta(x)
$$

The adsorption energy of one monomer at the interface is $\gamma k_{\mathrm{B}} T$ and the zero of energy is defined in such a way that $F_{\mathrm{sr}}=0$ when the concentration of monomers at the interface is the same as in the reservoir. We consider here only the case of reversible adsorption where the adsorbed polymer chains can freely exchange with the bulk solution that imposes the chemical potentials of the various components. Within the framework of the mean-field approximation, the total free energy $F_{\text {tot }}=F_{\text {ent }}+F_{\text {pol }}+F_{\text {elec }}+F_{\text {sr }}$ must be minimized with respect to the concentration profiles $\delta n(\mathbf{r}), \delta \phi_{\mathbf{i}}(\mathbf{r})$ and $\delta \phi(\mathbf{r})$. The expression of the electric field $\mathbf{E}(\mathbf{r})$ as a function of these profiles being unknown, we introduce a Lagrange multiplier $V(\mathbf{r})$ ensuring that the Maxwell equations are satisfied; this results in a new contribution to the free energy

$$
F_{\mathrm{Lagr}}=-\int \mathrm{d} \mathbf{r} V(\mathbf{r})[\varepsilon \nabla \cdot \mathbf{E}(\mathbf{r})-\rho(\mathbf{r})]
$$

where $\rho(\mathbf{r})$ is the charge density, $\rho(\mathbf{r})=-Z e\left(f \delta \phi(\mathbf{r})+\delta n(\mathbf{r})-\delta \phi_{\mathrm{i}}(\mathbf{r})\right)$, except at the surfaces where $\rho(\mathbf{r})=\sigma \delta(x)=\varepsilon \mathbf{E}(\mathbf{r}) \delta(x), \sigma$ being the surface charge density. In addition to the other variables, the minimization of the total free energy $F_{\text {tot }}+F_{\text {Lagr }}$ has also to be carried out with respect to the electric field $\mathbf{E}(\mathbf{r})$.

The minimization, combined with Maxwell equations, yields the Poisson-Boltzmann equations together with their boundary conditions, as given in reference [4]. The Lagrange multiplier $V(\mathbf{r})$ is the electrostatic potential and we rescale it as $\tilde{V}(\mathbf{r}) \equiv Z e \mathrm{~V}(\mathbf{r}) /\left(k_{\mathrm{B}} T\right)$.

As the non linear Poisson-Boltzmann equations are difficult to handle analytically, as a first step, throughout this paper, we study the weak adsorption limit where the fluctuations are small: $\delta n(\mathbf{r}) \ll n_{\mathrm{b}}, \delta \phi_{1}(\mathbf{r}) \ll \phi_{\mathrm{ib}} . \delta \phi(\mathbf{r}) \ll \phi_{\mathrm{b}}$ and $\tilde{V}(\mathbf{r}) \ll 1$. The linear Poisson-Boltzmann equations (Debye-Hückel equations) read

$$
\begin{aligned}
\delta n(\mathbf{r}) & =n_{\mathrm{b}} \tilde{V}(\mathbf{r}) \\
\delta \phi_{\mathrm{i}}(\mathbf{r}) & =-\phi_{\mathrm{ib}} \tilde{V}(\mathbf{r}) \\
\nabla^{2} \tilde{T}(\mathbf{r}) & =\kappa^{2} \tilde{V}(\mathbf{r})+4 \pi l_{\mathrm{B}} f \delta \phi(\mathbf{r}) \\
\Gamma^{2}(\delta \phi(\mathbf{r})) & =-12 \frac{f}{a^{2}} \phi_{\mathrm{b}} \tilde{V}(\mathbf{r})+\frac{12 \tilde{v} \phi_{\mathrm{b}}}{a^{2}} \delta \phi(\mathbf{r})
\end{aligned}
$$

Equations $(11,12)$ are simply linearized Boltzmann equations for ions in a Coulomb potential $V(\mathbf{r})$. Equations $(13,14)$ form a system of two coupled differential equations for the monomer concentration and the electric potential. The interaction of the solution with the interface is taken into account through the boundary conditions $\nabla \delta \phi\left(x=0^{+}\right)=-12 \gamma \phi_{\mathrm{b}} / a$ and $\nabla \tilde{V}(x=$ $\left.0^{+}\right)=-4 \pi l_{\mathrm{B}} \sigma /(Z e)$.

It should also be mentioned that the linearized mean field theory used here is entirely equivalent to considering the walls as creating a small external potential on the solutions and then using linear response theory. The linear response calculation is easily performed in the momentum variables, using the structure factor matrix as the response function [10].

3.2. Concentration Profiles and Electrostatic Potential. - The linear DebyeHückel equations are readily solved analytically. This leads to introduce two characteristic 
length scales of the concentration profiles $\lambda$ and $\zeta$ defined as

$$
\begin{aligned}
\frac{1}{\lambda} & =\frac{1}{\sqrt{2}} \sqrt{-\frac{1}{2}\left(\kappa^{2}+\frac{12 \tilde{v} \phi_{\mathrm{b}}}{a^{2}}\right)+\sqrt{\frac{12 \tilde{v} \phi_{\mathrm{b}} \kappa^{2}}{a^{2}}+q^{* 4}}} \\
\frac{1}{\zeta} & =\frac{1}{\sqrt{2}} \sqrt{\frac{1}{2}\left(\kappa^{2}+\frac{12 \tilde{v} \phi_{\mathrm{b}}}{a^{2}}\right)+\sqrt{\frac{12 \tilde{v} \phi_{\mathrm{b}} \kappa^{2}}{a^{2}}+q^{* 4}}}
\end{aligned}
$$

We first consider the case where both $\lambda$ and $\zeta$ are real positive. In Figure $1 \mathrm{~b}$, the region of the phase diagram where this occurs is shaded. This region is delimited by the line $\lambda^{-1}=0$ (lines b and $b^{\prime}$ in Fig. 1b) and by the limit of stability of the solution at finite wave vector (line $\mathrm{a}^{\prime}$ in Fig. 1b), where the length $\zeta$ diverges. Note that this region is different from the region where the polymer-polymer structure factor has a peak $\left(q^{*}>\kappa\right)$ shown in Figure 1a.' In all this region, the various concentration profiles show damped oscillations of period $2 \pi \lambda$, damped over a length $\zeta . \lambda<\zeta$ when $12 \tilde{v} \phi_{\mathrm{b}} /\left(\kappa^{2} a^{2}\right)>-1$ and $\lambda>\zeta$ when $12 \tilde{v} \phi_{\mathrm{b}} /\left(\kappa^{2} a^{2}\right)<-1$.

In the linear approximation that we use, the concentration profiles can be expressed as linear combinations of the two functions,

$$
\begin{aligned}
& A(x)=\frac{1}{2} \exp \left(-\frac{x}{\zeta}\right) \sin \left(\frac{x}{\lambda}\right) \\
& B(x)=\frac{1}{2} \exp \left(-\frac{x}{\zeta}\right) \cos \left(\frac{x}{\lambda}\right)
\end{aligned}
$$

In the low salt limit where the monomer-monomer structure factor shows a peak $q^{*} \gg \kappa$, well inside the oscillating concentration region (around number 4 in Fig. 1b), the two length scales $\lambda$ and $\zeta$ are equal to $2^{1 / 2} q^{*-1}$ On the border of this region, the period of the oscillations $\lambda$ diverges and the concentration profiles decay exponentially. The divergence of the damping length $\zeta$ on the stability line leads to periodic concentration profiles and thus to the formation of a lamellar phase of period $\left(q^{* 2}-\kappa^{2}\right)^{-1 / 2} \approx q^{*-1}$ induced by the interaction with the surface. As $q^{*}$ decreases along the line of instability $a^{\prime}$, the period increases and diverges at the Lifshitz point where the two length scales are infinite. Note again that we determine here the spinodal for the apparition of this phase and not the phase boundary. In the limit of high salt $q^{*} \ll \kappa$ (around number 3 in Fig. 1b), the damping length $\zeta$ is of order $\kappa^{-1}$ and the period of the oscillations $2 \pi \lambda$ is much larger and diverges on the boundary of the oscillatory region.

Inside the region where the concentration profiles are oscillating, the concentration profiles and the electrostatic potentials can be calculated explicitly. In the linear theory, they are expressed as a sum of a term proportional to $\gamma$ and a term proportional to $\sigma, \delta \phi(\mathbf{r})=\delta \phi_{\mathrm{el}}(\mathbf{r})+$ $\delta \phi_{\mathrm{sr}}(\mathbf{r})$ and $\tilde{V}=\tilde{V}_{\mathrm{el}}(\mathbf{r})+\tilde{V}_{\mathrm{sr}}(\mathbf{r})$, with

$$
\begin{aligned}
\delta \phi_{\mathrm{el}}(\mathbf{r}) & =\frac{q^{* 4}}{f} \frac{\sigma / Z e}{\zeta^{-2}+\lambda^{-2}}(\lambda A(x)+\zeta B(x)) \\
\delta \phi_{\mathrm{sr}}(\mathbf{r}) & =12 \frac{\gamma \phi_{\mathrm{b}}}{a}\left(-\left(1-\frac{\kappa^{2}}{\zeta^{-2}+\lambda^{-2}}\right) \lambda A(x)+\left(1+\frac{\kappa^{2}}{\zeta^{-2}+\lambda^{-2}}\right) \zeta B(x)\right) \\
\tilde{V}_{\mathrm{el}}(\mathbf{r}) & =4 \pi l_{\mathrm{B}} \frac{\sigma}{Z e}\left(-\left(1-\frac{12 \tilde{v} \phi_{\mathrm{b}} / a^{2}}{\zeta^{-2}+\lambda^{-2}}\right) \lambda A(x)+\left(1+\frac{12 \tilde{v} \phi_{\mathrm{b}} / a^{2}}{\zeta^{-2}+\lambda^{-2}}\right) \zeta B(x)\right) \\
\tilde{V}_{\mathrm{sr}}(\mathbf{r}) & =-\frac{q^{* 4}}{f} \frac{\gamma a}{\zeta^{-2}+\lambda^{-2}}(\lambda A(x)+\zeta B(x))
\end{aligned}
$$

As it appears from equations $(19-22), \delta \phi_{\mathrm{el}}(\mathbf{r}) \propto \tilde{V}_{\mathrm{sr}}(\mathbf{r})$. A simple explanation of this result is given in the next section. Note also that the correlation functions of the bulk polyelectrolyte 
solution ( $c f$. Sect. 2) can easily be obtained in real space by performing the Fourier transform of the expressions given by the matrix $S(\mathbf{q})$ (see $\mathrm{Eq} .(8)$ ). They are found to show oscillations in the same region as the concentration profiles and the potential, namely when $\lambda$ is a real number (see Fig. 1b).

Outside the oscillatory region delimited by the line $\lambda^{-1}=0$ (lines $b$ and $b^{\prime}$ ), the period $\lambda$ is imaginary and the electrostatic potentials can be written as the sum of exponentially decaying functions. The relevant length scales for the concentration profiles are then $\eta_{1}$ and $\eta_{2}$ defined by $\eta_{1}^{-1} \equiv \zeta^{-1}-i \lambda^{-1}$ and $\eta_{2}^{-1} \equiv \zeta^{-1}+i \lambda^{-1}$. The concentrations can be written as linear combinations of $C(x) \equiv \exp \left(-x / \eta_{1}\right)$ and $D(x) \equiv \exp \left(-x / \eta_{2}\right)$. In the high salt limit (around number 1 in Fig. 1b) where the correlation length of the equivalent neutral solution $\xi$ is larger than the Debye-Hïckel screening length $\kappa^{-1}$ and $q^{*} \ll \kappa$, the solution essentially behaves as a neutral polymer solution with an effective excluded volume $v^{\prime}=\tilde{v}+q^{* 4} a^{2} /\left(12 \kappa^{2} \phi_{\mathrm{b}}\right)$ where the second term $v_{\mathrm{el}}=q^{* 4} a^{2} /\left(12 \kappa^{2} \phi_{\mathrm{b}}\right)=4 \pi l_{\mathrm{B}} f^{2} / \kappa^{2}$ is an electrostatic contribution. The correlation length of the solution $\xi^{\prime}$ is then given by $\xi^{\prime-2}=3 v^{\prime} \phi_{\mathrm{b}} / a^{2}$. The bulk instability of the solution in this domain of the phase diagram is a macroscopic instability at zero wave vector. It occurs when the total excluded volume $v^{\prime}$ (including the electrostatic contribution) vanishes (line a in Fig. 1). On this instability line, the solution correlation length $\xi^{\prime}$ diverges. The two decay lengths $\eta_{1}$ and $\eta_{2}$ are such that $\eta_{1} \eta_{2}=\xi^{\prime} /(2 \kappa)$, and are equal on the line delimiting the oscillatory region. The decay length $\eta_{2}$ is equal to the screening length $\kappa^{-1}$ and $\eta_{1}$ is the solution correlation length $\xi^{\prime} / 2$; the range of the concentration perturbation introduced by the surface thus diverges on the spinodal line as expected. In the high excluded volume limit (around number 2 in Fig. 1b) where $\xi \ll \kappa^{-1}$ and $\xi \ll q^{*-1}$, the decay length $\eta_{2}=\xi / 2$ is the correlation length of the equivalent neutral polymer solution and $\eta_{1}=\xi^{\prime} /(\kappa \xi)$, is smaller than $\eta_{2}$. In most cases. $\eta_{1} \approx \kappa^{-1}$, since $\left(\xi / \xi^{\prime}\right)^{2}-1=\left(q^{*} / \kappa\right)^{2}\left(q^{*} \xi / 2\right)^{2}$ (and $\left.q^{*} \xi / 2 \ll 1\right)$.

The explicit concentration and electrostatic potential profiles in this region are:

$$
\begin{aligned}
\delta \phi_{\mathrm{el}}(\mathbf{r}) & =\frac{q^{* 4}}{f} \frac{\sigma}{Z e} \frac{1}{\eta_{2}^{-2}-\eta_{1}^{-2}}\left(\eta_{1} C(x)-\eta_{2} D(x)\right) \\
\delta \phi_{\mathrm{sr}}(\mathbf{r}) & =12 \frac{\gamma \phi_{\mathrm{b}}}{a} \frac{1}{\eta_{2}^{-2}-\eta_{1}^{-2}}\left(\left(\kappa^{2}-\eta_{1}^{-2}\right) \eta_{1} C(x)-\left(\kappa^{2}-\eta_{2}^{-2}\right) \eta_{2} D(x)\right) \\
\tilde{V}_{\mathrm{el}}(\mathbf{r}) & =4 \pi l_{\mathrm{B}} \frac{\sigma}{Z e} \frac{1}{\eta_{2}^{-2}-\eta_{1}^{-2}}\left(-\left(\kappa^{2}-\eta_{2}^{-2}\right) \eta_{1} C(x)+\left(\kappa^{2}-\eta_{1}^{-2}\right) \eta_{2} D(x)\right) \\
\tilde{V}_{\mathrm{sr}}(\mathbf{r}) & =-\frac{q^{* 4}}{f} \frac{\gamma a}{\eta_{2}^{-2}-\eta_{1}^{-2}}\left(\eta_{1} C(x)-\eta_{2} D(x)\right)
\end{aligned}
$$

Note that even in this range of parameters the concentration profiles are not always monotonic due to the possible antagonistic effects of the electrostatic and short-range interactions. The polymer can carry the same electrostatic charge as the surface and still be attracted because of the short-range interactions, which impose a boundary condition on the concentration gradient of monomers at the interface. In the limit where the fraction of charged monomers is very small $\left(q^{*} \rightarrow 0\right)$ the electrostatic interactions are decoupled from the short range interactions and $\delta \phi_{\mathrm{el}}(\mathbf{r}) \ll \delta \phi_{\mathrm{sr}}(\mathbf{r})$ and $\tilde{V}_{\mathrm{sr}}(\mathbf{r}) \ll \tilde{V}_{\mathrm{el}}(\mathbf{r}) ;$ we then find $\delta \phi_{\mathrm{sr}}(x) \propto \exp (-2 x / \xi)$ and $V_{\mathrm{el}}(\mathbf{r}) \propto$ $\exp (-\kappa x)$, which corresponds to the well established results for the weak adsorption of a neutral polymer at an interface (within the self-consistent field method) [9] and to the screening of a charged surface by ions (Debye-Hückel theory) [11]. 


\section{Polyelectrolyte Solution Between Two Surfaces}

4.1. Concentration Profiles and Electrostatic Potential. - We now consider a polyelectrolyte solution in a slab of thickness $L$ between two surfaces. The solution in the gap is as before in equilibrium with a reservoir that imposes the chemical potentials of the various constituents. Each surface carries a charge $\sigma$ per unit area and has a short-ranged interaction parameter $\gamma$ with the monomers. This would constitute an idealized model for the interaction between two charged colloidal particles immersed in a polyelectrolyte solution. We first evaluate the concentration profiles in the gap between the two surfaces and then we study the interaction between the two surfaces mediated by the polyelectrolyte solution.

The $z$-axis is defined along the perpendicular to the two surfaces located respectively at $z=-L / 2$ and $z=+L / 2$ (see Fig. 2b). The adsorption (or depletion) on one surface is affected by the interaction with the other interface. This effect is noticeable as long as surfaces are not too far apart. More precisely, if the distance from the interface located at $z=-L / 2$ is denoted by $x=z+L / 2$. the results obtained for the adsorption (depletion) on a single interface are obtained in the limit $L \rightarrow \infty$ at fixed $x$.

Technically, the method used for the adsorption on a single surface are generalized straightforwardly. The only difference in the Debye-Hückel equations for the concentration profiles and the electrostatic potential comes from the boundary conditions, the gradients of the potential $\tilde{V}(\mathbf{r})$ and of the monomer concentration profile $\phi(\mathbf{r})$ being fixed for $z=-L / 2$ as well as for $z=+L / 2$. The resolution of the Debye-Hückel equations leads to the same expressions obtained in equations (19-22) and equations (23-26), but with different expressions for $A(x)$, $B(x), C(x)$ and $D(x)$ that depend on the distance $L$ between the surfaces. In terms of the variable $z$,

$$
\begin{aligned}
A_{L}(z)= & \frac{1}{\cosh (L / \zeta)-\cos (L / \lambda)}\left(\sin \left(\frac{L}{2 \lambda}\right) \cosh \left(\frac{L}{2 \zeta}\right) \cos \left(\frac{z}{\lambda}\right) \cosh \left(\frac{z}{\zeta}\right)\right. \\
& \left.-\cos \left(\frac{L}{2 \lambda}\right) \sinh \left(\frac{L}{2 \zeta}\right) \sin \left(\frac{z}{\lambda}\right) \sinh \left(\frac{z}{\zeta}\right)\right) \\
B_{L}(z)= & \frac{1}{\cosh (L / \zeta)-\cos (L / \lambda)}\left(\sin \left(\frac{L}{2 \lambda}\right) \cosh \left(\frac{L}{2 \zeta}\right) \sin \left(\frac{z}{\lambda}\right) \sinh \left(\frac{z}{\zeta}\right)\right. \\
& \left.+\cos \left(\frac{L}{2 \lambda}\right) \sinh \left(\frac{L}{2 \zeta}\right) \cos \left(\frac{z}{\lambda}\right) \cosh \left(\frac{z}{\zeta}\right)\right) \\
C_{L}(z)= & \frac{\cosh \left(z / \eta_{1}\right)}{\sinh \left(L /\left(2 \eta_{1}\right)\right)} \\
D_{L}(z)= & \frac{\cosh \left(z / \eta_{2}\right)}{\sinh \left(L /\left(2 \eta_{2}\right)\right)}
\end{aligned}
$$

The characteristics of these concentration profiles are the same as for the adsorption on a single interface. Damped oscillatory profiles are observed in the same region of the phase diagram with a characteristic length scale given either by the peak of the bulk structure factor $\left(q^{*-1}\right)$ or by $\kappa^{-1}$ when the structure factor does not show a peak. In the other regions of the phase diagram, the concentrations decay exponentially from the surfaces over the two characteristic length scales $\eta_{1}$ and $\eta_{2}$.

\subsection{Interaction Between Surfaces Mediated by a Polyelectrolyte Solution.} - The grand canonical free energy of the polyelectrolyte solution in the gap cau be obtained directly from the concentration profiles by integration of the free energy density. An other and 
convenient way to calculate this energy is to consider it as a function of the following independent thermodynamic variables: temperature, chemical potentials of the various constituents, surface charge $\sigma$ and interaction parameter $\gamma$. At constant value of the temperature and of the chemical potentials, the differential of the free energy per unit area $g=F / S$ ( $S$ being the total area of each surface) can be written as

$$
\mathrm{d} g=2 V(z=L / 2) \mathrm{d} \sigma-2 k_{\mathrm{B}} T a \delta \phi(z=L / 2) \mathrm{d} \gamma
$$

where the factor 2 accounts for the existence of the two surfaces. The electrostatic potential on one surface $I^{-}(z=L / 2)$ and the surface concentration $\delta \phi(z=L / 2)$ are in the DebyeHückel theory linear combinations of $\gamma$ and $\sigma$. The free energy per unit area is obtained by integration as

$$
g=\sigma V_{\mathrm{el}}(z=L / 2)-k_{\mathrm{B}} T \gamma a \delta \phi_{\mathrm{sr}}(z=L / 2)+2 \sigma V_{\mathrm{sr}}(z=L / 2)
$$

Note that the Maxwell relations on the free energy impose that on the surfaces, $V_{\mathrm{sr}}^{r} \propto \delta \phi_{\mathrm{el}}$ as can be directly checked from the concentration profiles. The force between the surfaces per unit area (the pressure) is then calculated by derivation of the free energy: $P=-\frac{\partial g}{\partial L}=$ $-\sigma V_{\mathrm{el}}^{\prime}(z= \pm L / 2)+k_{\mathrm{B}} T \gamma a \delta \phi_{\mathrm{sr}}^{\prime}(z= \pm L / 2)-2 \sigma V_{\mathrm{sr}}^{\prime}(z= \pm L / 2)$, where the prime denotes a differentiation with respect to $L$.

In the regime where the concentration profiles show damped oscillations, the pressure, as a function of $L$, also shows damped oscillations with the same characteristic length scales. The derivatives of the surface potential and of the surface concentration in the expression of the force only involve derivatives of the functions $A_{L}(L / 2)$ and $B_{L}(L / 2)$ with respect to $L$.

$$
\begin{gathered}
A_{L}^{\prime}(L / 2)=\frac{-\lambda^{-1}\left(1-\cos (L / \lambda) \cosh (L / \zeta)+\lambda \zeta^{-1} \sin (L / \lambda) \sinh (L / \zeta)\right)}{2[\cosh (L / \zeta)-\cos (L / \lambda)]^{2}} \\
B_{L}^{\prime}(L / 2)=\frac{\zeta^{-1}\left(1-\cos (L / \lambda) \cosh (L / \zeta)-\zeta \lambda^{-1} \sin (L / \lambda) \sinh (L / \zeta)\right)}{2[\cosh (L / \zeta)-\cos (L / \lambda)]^{2}}
\end{gathered}
$$

The force decays as $\exp (-L / \zeta)$ in the limit where the distance $L$ between the plates is larger than the damping length $\zeta$.

Quite similarly, in the region where the concentration profiles are not oscillating, the force is expressed as a function of the derivatives of the two functions $C$ and $D$.

$$
\begin{aligned}
& C^{\prime}(L)=-\frac{\sinh ^{-2}\left(L /\left(2 \eta_{1}\right)\right)}{2 \eta_{1}} \\
& D^{\prime}(L)=-\frac{\sinh ^{-2}\left(L /\left(2 \eta_{2}\right)\right)}{2 \eta_{2}}
\end{aligned}
$$

The force decays exponentially if the distance $L$ is larger than the two characteristic length scales $\eta_{1}$ and $\eta_{2}$. The sign of the force strongly depends on the relative values of the various parameters in this case and the force can have a non monotonic variation with the distance. This is due to the competition between the electrostatic and non electrostatic interactions.

We give in Figure 3 typical force profiles in the various regions of the phase diagram. Figure $3 \mathrm{a}$ features the case of a system which shows oscillations, and corresponds to a point close the line of instability $\mathrm{a}^{\prime}$ in Figure 1 . The period of the oscillations is $2 \pi \lambda \approx 107.3 a$ and the damping length is $\zeta \approx 59.3 a$ ( $a$ being the size of a monomer). In the limit where the polymer is very weakly charged $(f \rightarrow 0)$, the electrostatic force and the force due to the polymer can 

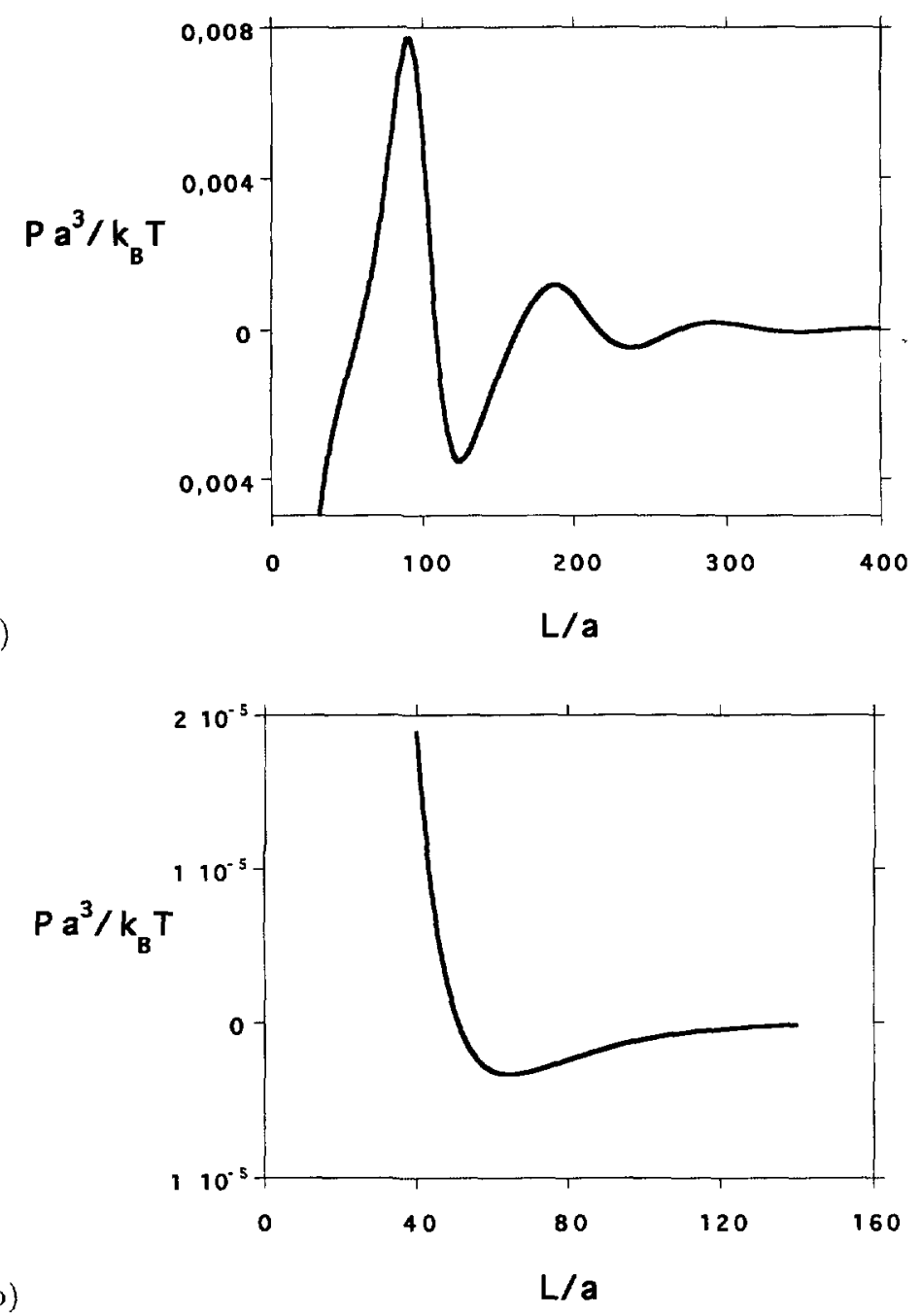

Fig. 3. - Normalized electrostatic pressure $P a^{3} /\left(k_{\mathrm{B}} T\right)$ between the two surfaces as a function of their separation $L / a$. The size of the monomers is $a=5 \AA$, and a fraction $f=0.01$ of them is charged. All charges are monovalent, $Z=1 . T=300 \mathrm{~K}$ and $\varepsilon=80$ (water), which sets the Bjerrum length $l_{\mathrm{B}}=6.942 \AA$. The electrostatic screening length is $\kappa^{-1}=44 \AA$, which corresponds to a bulk concentration of about $5 \times 10^{-3} \mathrm{M}$ and to $\kappa^{2} \approx 1.29 \times 10^{-2} \mathrm{a}^{-2}$. The surfaces are positively charged with a charge density $e / 2500 \AA^{-2}$ a) $\phi_{\mathrm{b}}=10^{-4} \AA^{-3}$ and $\tilde{v}=-16 \AA^{3}$, which corresponds to $q^{* 2} / \kappa^{2} \approx 1.26$ and $12 \tilde{v} \phi_{\mathrm{b}} /\left(\kappa^{2} a^{2}\right) \approx-1.49$. b) $\phi_{\mathrm{b}}=10^{-6} \AA^{-3}$ and $\tilde{v}=125 \AA^{3}$. which corresponds to $q^{* 2} / \kappa^{2} \approx 0.13$ and $4 /(\kappa \xi)^{2}=0.12$. c) $\phi_{\mathrm{b}}=10^{-4} \AA^{-3}$ and $\tilde{v}=125 \AA^{3}$, which corresponds to $q^{* 2} / \kappa^{2} \approx 1.26$ and $4 /(\kappa \xi)^{2}=11.6$.

simply be added. The electrostatic force is given by the standard Debye-Hückel theory; it is repulsive and decays over the Debye-Hïckel screening length. The force due to the polymer is attractive irrespective of the sign of the interaction $\gamma$ and has a range given by the correlation length $\xi / 2$. An example is given in Figure $3 \mathrm{~b}$, corresponding to region 1 in Figure $1 \mathrm{~b}$, with $\eta_{1} \approx 8.88 a \approx \kappa^{-1}$ and $\eta_{2} \approx 24.0 a \approx \xi / 2$. In Figure $3 c$, the pressure is again the sum of a 


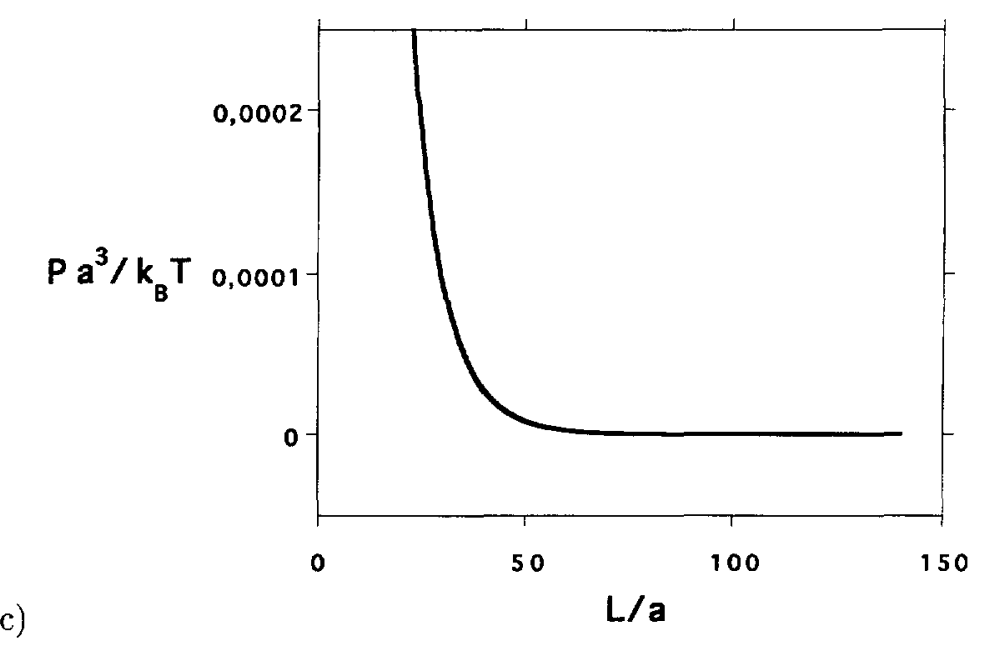

Fig. 3. - (Contınued.)

screened electrostatic repulsion decaying with a characteristic length $\eta_{1} \approx 8.21 a \approx \kappa^{-1}$ and a small attraction due to the polymer decaying faster, and thus not seen, with a characteristic length $\eta_{2} \approx 2.60 a \approx \xi / 2$.

\section{Surface Excess}

An important and experimentally relevant parameter is the polymer surface excess

$$
\Gamma \equiv \frac{1}{2} \int_{z=-L / 2}^{z=+L / 2} \mathrm{~d} z\left(\phi(z)-\phi_{\mathrm{b}}\right)=\frac{1}{2} \int_{z=-L / 2}^{z=+L / 2} \mathrm{~d} z \delta \phi(z)
$$

The surface excess can be obtained directly from the Debye-Hückel equations (11-14) without solving for the detailed concentration profile for example by analyzing them in Fourier components. The polymer surface excess $\Gamma$ has two contributions: one due to the electrostatic interaction with the interface $\Gamma_{\mathrm{el}}$ and another one due to short-ranged interactions $\Gamma_{\mathrm{sr}}$. In the linear approximation, these two contributions add up and $\Gamma=\Gamma_{\mathrm{el}}+\Gamma_{\mathrm{sr}}$.

$$
\begin{aligned}
\Gamma_{\mathrm{el}} & =\frac{\sigma}{Z e} \frac{q^{* 4} / f}{12 \tilde{v} \phi_{\mathrm{b}} \kappa^{2} / a^{2}+q^{* 4}}=\frac{\sigma}{Z e} \frac{1}{\left(n_{\mathrm{b}}+\phi_{\mathrm{ib}}\right) \tilde{v} / f+f} \\
\Gamma_{\mathrm{sr}} & =\frac{12 \phi_{\mathrm{b}} \gamma / a}{12 \tilde{v} \phi_{\mathrm{b}} / a^{2}+q^{* 4} / \kappa^{2}}=\frac{\gamma a}{\tilde{v}+f^{2} /\left(n_{\mathrm{b}}+\phi_{\mathrm{ib}}\right)}
\end{aligned}
$$

In the weak adsorption limit that we are studying, the polymer surface excess is independent of the distance between the interacting surfaces $L$. It also depends only very weakly on the bulk polymer concentration through the effective excluded volume parameter $\tilde{v}$ and the inverse Debye-Hückel screening length $\kappa$. We now discuss its variation with the ionic strength and with the fraction of charged monomers $f$.

We consider for example the case of adsorption $\left(\Gamma_{\mathrm{el}}, \Gamma_{\mathrm{sr}}>0\right)$. The addition of salt to the solution has two opposing effects. On the one hand, there is a repulsion inside the adsorbed layer between the charged monomers and the addition of salt results in a more efficient screening of these interactions and consequently in a stronger adsorption. This effect is present in 
equation (39) for $\Gamma_{\mathrm{sr}}$. On the other hand, if the interface is charged, there is also a competition between the salt molecules and the charged monomers for the adsorption at the interface, favoring a smaller polymer adsorbed amount (this can be also viewed as a screening of the polymer surface electrostatic interactions). The dominant effect is not obvious and experiments have reported different results for the dependence of the adsorbed polymer amount as a function of the salt concentration [12-15]. Equation (38) predicts that whenever the effective excluded volume parameter $\tilde{v}$ is positive, the latter effect is dominant and the adsorption of polyelectrolyte at a charged interface decreases upon addition of salt. On the contrary, when $\tilde{v}$ is negative, monomers attract each other and $\Gamma_{\text {el }}$ increases with the salt concentration; the screening of the electrostatic repulsion between the charged monomers has a drastic effect and even leads eventually to an instability in the solution, $\Gamma \rightarrow \infty$ corresponding to a wetting transition of the interface by the polyelectrolyte (See also Fig. 1a). Note that equations $(38,39)$ suggest that the instability occurs at $\tilde{v}=-f^{2} /\left(n_{\mathrm{b}}+\phi_{\mathrm{ib}}\right)$, which corresponds to the instability at wavevector $q=0$ mentioned in Section 2. In fact, when the structure factor diverges at a wavevector $q \neq 0$, at the instability, the total adsorbed amount remains finite.

The dependence of the polymer surface excess with the fraction of charged monomers $f$ is also complex: the repulsions inside the adsorbed layer increase with $f$ and oppose adsorption, as appears in equation (39) while the attraction towards an oppositely charged interface increases with $f$. When the excluded volume $v$ is positive, $\Gamma_{\text {el }}$ shows a maximum as a function of $f$ for $f^{2}=\tilde{v}\left(n_{\mathrm{b}}+\phi_{\mathrm{bb}}\right)$, as long as $\tilde{v}\left(n_{\mathrm{b}}+\phi_{\mathrm{ib}}\right)<1$. This effect was already found both experimentally $[14-17]$ and theoretically $[18,19]$. It should be noted, however, that the total adsorbed charge $Z$ ef $\Gamma$ increases monotonically with $f$. When $\tilde{v}$ is negative. the repulsion between charged monomers is always the dominant effect, even at a low fraction of charged monomers, and $\Gamma_{\mathrm{el}}$ as well as $f \Gamma_{\mathrm{el}}$ are decreasing functions of $f$.

In the case where $\gamma$ and $\sigma$ are of opposite signs, the total polymer surface excess vanishes when $\sigma f /(Z e)=-\gamma a\left(n_{\mathrm{b}}+\phi_{\mathrm{ib}}\right)$. This result is independent of the excluded volume and thus of the solvent quality. The neutral attraction necessary to compensate an electrostatic repulsion decreases as the inverse of the ionic strength.

\section{Discussion and Conclusions}

We have shown in this paper that the tendency of a polyelectrolyte solution to form mesophases at low salt concentration leads to oscillations in the concentration profiles (for the monomers and for the ions) in the vicinity of an adsorbing surface even if the bulk solution remains homogeneous. The region of the phase diagram where the oscillatory profiles are predicted is different from the region where the monomer-monomer structure factor shows a peak in momentum space, but corresponds to the region where it has oscillations in the real space. When the salt concentration is small and not too close to lines $a^{\prime}$ and $b$ of Figure $1 b$, the period of the oscillations and the decay length of the oscillation amplitudes are of the same order of magnitude and are proportional to the characteristic length scale of the solution given by the position of the peak of the structure factor $\left(q^{*-1}\right)$ when the structure factor shows a peak. In the vicinity of the instability of the bulk solution with respect to mesophase formation, the decay length diverges and an ordered lamellar phase wets the adsorbing surface. We have in this work considered only the stability limit of the various phases and we have not determined precisely the phase boundaries and in particular the wetting transition.

In the same region of the phase diagram, the force between charged surfaces immersed in the polyelectrolyte solution also shows oscillations with the distance between the surfaces with the same characteristic length scales. Theoretical models based on the so-called random phase approximation such as the one proposed here seem to describe reasonably well the properties 
of weakly charged polyelectrolytes such as polyacrylic acid in a concentrated solution. It would be interesting to test quantitatively the predictions for the force by direct measurements with a surface force apparatus.

In the other regions of the phase diagram, both the concentration profiles and the force between surfaces decay exponentially and can be written as the sum of two exponentials. In this case the force is not always monotonic because of the competing effects of the electrostatic and non electrostatic interactions. In the limit of high salt concentration, the interaction force is the sum of the repulsive Debye-Hückel force and of an attractive force due to the quasi-neutral polymer.

In our simple model, the polymer surface excess between two surfaces is independent of the distance between them. Its contribution due to electrostatic interactions has a maximum when the fraction of charged monomers is $f=\sqrt{\tilde{v}\left(n_{\mathrm{b}}+\phi_{\mathrm{ib}}\right)}$ (for $\tilde{v}>0$ ).

The main assumption on which all the results presented in this paper rely is the weak adsorption approximation which allows to consider that the change in concentration due to the interface is small. This requires a small charge of the interface and a weak non electrostatic interaction between the monomers and the surface. Although this is a rather extreme approximation, it should at least give a qualitative picture of polyelectrolyte adsorption and of the relevant length scales involved. In order to go beyond this linear assumption, one must solve numerically the Poisson-Boltzmann equations as done in reference [4]. A comparison of equations $(23,24)$ with Figure 1 of reference [4] shows a good qualitative agreement. We expect that oscillatory concentration profiles should also be obtained for values of the parameters falling outside of the range of validity of the weak adsorption approximation.

The other major assumption of our work is that the chains remain Gaussian and thus that the electrostatic interactions remain small and do not stiffen the chains locally as expected in a dilute solution. We expect this approximation to be reasonable for weakly charged polyelectrolytes at high enough concentrations.

One of the main conclusions of our work is the very rich variety of behaviors that can be expected for polyelectrolyte adsorption due to the large number of relevant parameters. In this respect, our approach is also oversimplified. We have throughout the paper considered that the small ions, salt or counterions are pointlike. They can in many cases have specific interactions with both the polymer and the charged surfaces. If for example the salt ions adsorb on the interface they can displace the polymer and prevent polymer adsorption. For multivalent ions there are also strong charge correlations which are not adequately described by the Debye-Hückel theory. The solvent has been treated as a continuum with a well defined dielectric constant; this could be a poor approximation in the vicinity of an interface where the polymer concentration can become high.

The polyelectrolyte chains have been considered as quenched in the sense that they have a fixed charge uniformly distributed along the chains and that the charged fraction $f$ is constant. For polyacids or polybases the charge is not quenched but annealed. The average charge is fixed by a chemical equilibrium between the neutral and charged forms of the monomers and can be controlled by tuning the $\mathrm{pH}$ of the bulk solution. In the vicinity of a charged surface, the fraction of charged monomers varies because of the electrostatic interaction with the surface and the screening is increased by the polarisability of the polymer charges. The case of annealed polyelectrolytes is treated in details in the appendix.

\section{Acknowledgments}

We are grateful to A. Johner and I. Borukhov for helpful discussions. 


\section{Appendix}

\section{Annealed Polyelectrolytes}

In contrast to quenched polyelectrolyte chains, where the fraction of charged monomers $f$ is fixed, annealed polyelectrolyte chains can adjust their fraction of charged monomers locally. For instance, if the monomers have an acidic group A-H (e.g. carboxylic groups in polyacrylic acid), the associated chemical reaction is

$$
\mathrm{AH} \rightleftharpoons \mathrm{A}^{-}+\mathrm{H}^{+}
$$

The fraction $f$ of charged monomers $\mathrm{A}^{-}$is related to the concentration $c_{\mathrm{H}^{+}}$of $\mathrm{H}^{+}$ions by the law of mass action

$$
\frac{f}{1-f}=\frac{K_{\mathrm{D}}}{c_{\mathrm{H}^{+}}}
$$

where $K_{\mathrm{D}}$ is the dissociation constant. For simplicity, we assume in the following that the valency of all charges is $Z=1$ and that the charged monomers carry a negative charge.

In our model, the free energy of an annealed polyelectrolyte solution is composed of the same terms as in equation (1). But $F_{\text {ent }}$ and $F_{\text {pol }}$ are slightly modified, and we denote them by $F_{\text {ent }}^{\text {a }}$ and $F_{\text {pol }}^{\mathrm{a}}$. The entropy of mixing of the ions $\mathrm{H}^{+}$and $\mathrm{OH}^{-}$has to be taken into account in $F_{\text {ent }}^{\mathrm{a}}$, in addition to those of the counter-ions and of the salt. The polymer free energy of the annealed chains also has an additional term due to the entropy of mixing of the charges along the chains

$$
\begin{aligned}
F_{\mathrm{pol}}^{\mathrm{a}}= & k_{\mathrm{B}} T \int \mathrm{d} \mathbf{r}\left[\frac{a^{2}}{24 \phi(\mathbf{r})}|\nabla \phi|^{2}(\mathbf{r})+\frac{1}{2} v\left(\phi^{2}(\mathbf{r})-\phi_{\mathrm{b}}^{2}\right)+\frac{1}{6} w^{2}\left(\phi^{3}(\mathbf{r})-\phi_{\mathrm{b}}^{3}\right)\right. \\
& -\mu\left(f(\mathbf{r}) \phi(\mathbf{r})-f_{0} \phi_{\mathrm{b}}\right)-\nu\left((1-f(\mathbf{r})) \phi(\mathbf{r})-\left(1-f_{0}\right) \phi_{\mathrm{b}}\right) \\
& +\phi(\mathbf{r})(f(\mathbf{r}) \log (f(\mathbf{r}))+(1-f(\mathbf{r})) \log (1-f(\mathbf{r}))) \\
& \left.-\phi_{\mathrm{b}}\left(f_{0} \log \left(f_{0}\right)+\left(1-f_{0}\right) \log \left(1-f_{0}\right)\right)\right]
\end{aligned}
$$

$\mu$ and $\nu$ are the reference chemical potentials of the charged and the neutral monomers respectively. $f_{0}$ is the fraction of charged monomers in the reservoir. The minimization of the free energy $F_{\text {pol }}^{\mathrm{a}}$ with respect to the concentrations of charged and neutral monomers (or. equivalently, with respect to $f(\mathbf{r})$ and $\phi(\mathbf{r}))$ when $f(\mathbf{r})=f_{0}$ and $\phi(\mathbf{r})=\phi_{\mathrm{b}}$ yields the values of the reference chemical potentials $\mu=v \phi_{\mathrm{b}}+w^{2} \phi_{\mathrm{b}}^{2} / 2+\log \left(f_{0}\right)$ and $\nu=v \phi_{\mathrm{b}}+w^{2} \phi_{\mathrm{b}}^{2} / 2+\log \left(1-f_{0}\right)$. Substituting in equation (A.3), we finally obtain

$$
\begin{aligned}
F_{\text {pol }}^{\mathrm{a}}=k_{\mathrm{B}} T & \int \mathrm{d} \mathbf{r}\left[\frac{a^{2}}{24 \phi(\mathbf{r})}|\nabla \phi|^{2}(\mathbf{r})+\frac{1}{2} \tilde{v}\left(\phi(\mathbf{r})-\phi_{\mathrm{b}}\right)^{2}\right. \\
& \left.+\phi(\mathbf{r})\left(f(\mathbf{r}) \log \left(\frac{f(\mathbf{r})}{f_{0}}\right)+(1-f(\mathbf{r})) \log \left(\frac{1-f(\mathbf{r})}{1-f_{0}}\right)\right)\right]
\end{aligned}
$$

$\tilde{v}=v+w^{2}\left(\phi(\mathbf{r})+2 \phi_{\mathrm{b}}\right) / 3$ is the effective excluded volume. as defined in Section 2 .

In a mean-field approximation, the minimization of the total free energy $F_{\text {tot }}^{\mathrm{a}}$ has to be carried out with respect to $n(\mathbf{r}), \phi_{1}(\mathbf{r}), \phi(\mathbf{r}), c_{\mathrm{H}^{+}}(\mathbf{r}), c_{\mathrm{OH}^{-}}(\mathbf{r}), E(\mathbf{r})$ and $f(\mathbf{r})$. The seven PoissonBoltzmann equations include four trivial Boltzmann equations for the ions concentration; for instance, the concentration of protons is given by

$$
c_{\mathrm{H}^{+}}(\mathbf{r})=c_{\mathrm{H}^{+}}^{\mathrm{b}} \exp (-\tilde{V}(\mathbf{r}))
$$


where the concentration of ions $\mathrm{H}^{+}$in the reservoir is denoted by $c_{\mathrm{H}^{+}}^{\mathrm{b}}$. The minimization with respect to $f(\mathbf{r})$ yields the law of mass action with a dissociation constant $K_{\mathrm{D}}=c_{\mathrm{H}^{+}}^{\mathrm{b}} \exp (\mu-\nu)$

$$
\frac{f(\mathbf{r})}{1-f(\mathbf{r})}=\frac{f_{0}}{1-f_{0}} \exp (\tilde{V}(\mathbf{r}))=\frac{f_{0}}{1-f_{0}} \frac{c_{\mathrm{H}^{+}}^{\mathrm{b}}}{c_{\mathrm{H}^{+}}(\mathbf{r})}
$$

The last two equations constitute a coupled system of two differential equations for $\phi(\mathbf{r})$ and $\tilde{V}(\mathbf{r})$, and were obtained in reference [4]. After linearization, a system of two coupled linear differential equations for $\delta \phi(\mathbf{r})$ and $\tilde{V}(\mathbf{r})$ is obtained, similar to equations $(13,14)$, the only difference being a decrease of the Debye-Hückel length $\kappa^{-1}$ which is given by

$$
\kappa^{2}=4 \pi l_{\mathrm{B}}\left(\left(n_{\mathrm{b}}+\phi_{\mathrm{ib}}+c_{\mathrm{H}^{+}}^{\mathrm{b}}+c_{\mathrm{OH}^{-}}^{\mathrm{b}}\right)+f_{0}\left(1-f_{0}\right) \phi_{\mathrm{b}}\right)
$$

This result was already mentioned in reference [20]. The Debye-Hückel length $\kappa^{-1}$ for an annealed polyelectrolyte solution is smaller than for a quenched solution since annealed charges are free to adjust to the local conditions and to screen the electrostatic interactions more efficiently.

The charge of the polymer also changes with the distance to the adsorbing surface. The charge fluctuation $\delta f=f-f_{0}$ is proportional to the reduced electrostatic potential $\tilde{V}(\mathbf{r})$.

\section{References}

[1] Napper D.H., Polymeric Stabilization of Colloidal Dispersions (Academic Press, London, 1983).

[2] Fleer G.J., Cohen Stuart M.A., Scheutjens J.M.H.M., Cosgrove T. and Vincent B., Polymers at Interfaces (Chapman \& Hall, London, 1993).

[3] Hesselink T.H., J. Colloid Interface Sci. 60 (1977) 448.

[4] Borukhov I., Andelman D. and Orland H., Europhys. Lett. 32 (1995) 499-504.

[5] Barrat J.L. and Joanny J.F., Adv. Chem. Phys. 44 (1996) 2-66.

[6] Borue V.Y. and Erukhimovich I.Y., Macromolecules 21 (1988) 3240-3249.

[7] Joanny J.F. and Leibler L., J. Phys. France 51 (1990) 545-557.

[8] Edwards S.F.. Proc. Phys. Soc. 85 (1965) 613-624; Edwards S.F., Proc. Phys. Soc. 88 (1966) 265

[9] de Gennes P.G., Scaling Concepts in Polymer Physics (Cornell University Press, Ithaca, 1979).

[10] The only difference is that, in the linear response theory, the minimization is carried out after the expansion of the free energy with respect to the small parameters, while equations (11-14) are obtained by first minimizing the free energy and then linearizing Poisson-Boltzmann equations.

[11] Israelachvili J., Intermolecular \& Surface Forces (Academic press, London, 1991).

[12] Papenhuijzen J., Fleer G.J. and Bijsterbosch B.H., J. Colloid Interface Sci. 104 (1985) $530-539$.

[13] Williams P.A., Harrop R., Phillips G.O., Pass G. and Robb I.D.. J. Chem. Soc., Faraday Trans. I. 78 (1982) 1733-1740.

[14] Durand G., Lafuma F. and Audebert R., Progr. Colloid Polym. Scı. 266 (1988) 278-282. 
[15] Popping B., Deratani A., Sebille B., Desbois N., Lamarche J.M. and Foissy A., Calloids Surfaces 64 (1992) 125-133.

[16] Wang T.K. and Audebert R., J. Colloid Interface Sci. 121 (1988) 32-41.

[17] Blaakmeer J., Böhmer M.R., Cohen Stuart M.A. and Fleer G.J., Macromolecules 23 (1990) 2301-2309.

[18] van de Steeg H.G.M., Cohen Stuart M.A., de Keizer A. and Bijsterbosch B.H., Langmurr 8 (1992) 2538-2546.

[19] Evers O.A., Fleer G.J., Scheutjens J.M.H.M. and Lyklema J., J. Colloid Interface Sci. 111 (1986) 446-454.

[20] Raphael E. and Joanny J.F., Europhys. Lett. 13 (1990) 623-628. 